\title{
Incidental Asymptomatic Splenic Infarct in a COVID-19 Patient
}

\author{
Natasha Ghalib ${ }^{1}$, Prateek Pophali ${ }^{1}$, Natalia Chamorro-Pareja ${ }^{1}$, Apoorva Jayarangaiah ${ }^{2}$, Abhishek Kumar
} 2

1. Internal Medicine, Albert Einstein College of Medicine/Jacobi Medical Center, Bronx, USA 2. Hematology/Oncology, Albert Einstein College of Medicine/Jacobi Medical Center, Bronx, USA

Corresponding author: Apoorva Jayarangaiah, jayarana@nychhc.org

\begin{abstract}
A high incidence of thromboembolic events and coagulation parameter abnormalities are seen in cases of coronavirus disease 2019 (COVID-19). Both venous and arterial thrombosis, including arterial thrombosis in unusual sites, have been reported in COVID patients in recent literature. Herein, we report a case of a 67year-old female patient with non-critical COVID-19 disease with an incidental finding of an asymptomatic splenic infarct. In the absence of a cardio-embolic source, we believe this was an arterial thromboembolic event in the splenic circulation. The duration and modality of anticoagulation of inpatient and ambulatory COVID patients remains a dynamic discussion. Our case adds the evidence of a clinically silent arterial thrombotic event in a non-critical COVID-19 patient which further emphasizes the need to address the strategies for diagnosis and management of thrombo-embolism to prevent potentially fatal complications.
\end{abstract}

Categories: Internal Medicine, Public Health, Hematology

Keywords: covid-19, splenic infarct, arterial thrombosis

\section{Introduction}

The United States, amongst other countries, has been severely affected by the coronavirus disease 2019 (COVID-19) pandemic caused by the severe acute respiratory syndrome coronavirus 2 (SARS-CoV-2). COVID-19 is primarily a respiratory disease with multisystem involvement and a high mortality rate, especially amongst hospitalized patients (21\%) [1]. Patients with COVID-19 infection harbor a hypercoagulable state as part of the disease spectrum [2]. This hypercoagulable state is due to the systemic inflammation induced by the elevated cytokines causing endothelial activation [3]. Another pathogenic mechanism for the pro-thrombotic state is activation of hypoxia-inducible transcription factors (HIFs) [4]. As we learn more about the biology of COVID-19, more pathogenic mechanisms for the procoagulant state are being reported. There are multiple reports of clinically significant thrombosis in critically ill patients [2,5-7]. Herein, we report a case of a non-critical COVID-19 patient admitted to a New York City public hospital with an incidental finding of splenic infarction.

Review began $12 / 28 / 2020$ Review ended 01/28/2021 Published 02/01/2021

\section{(๑) Copyright 2021}

Ghalib et al. This is an open access article distributed under the terms of the Creative Commons Attribution License CC-BY 4.0., which permits unrestricted use, distribution, and reproduction in any medium, provided the original author and source are credited.

\section{Case Presentation}

A 67-year-old female with a past medical history of hypertension, diabetes mellitus, coronary artery disease, hypothyroidism, and mild intermittent asthma was admitted to our hospital with a one-week history of progressively worsening shortness of breath, hypoxia, and fever. She also noted pain in her left calf for a similar duration without any erythema or edema. She denied recent hospitalization, surgery, trauma, or a history of clots. She had exposure from her mother, who recently passed away due to COVID-19. Her vitals on presentation were temperature $101 \mathrm{~F}$, heart rate 100 beats/min, blood pressure $127 / 76 \mathrm{mmHg}$, respiratory rate $45 / \mathrm{min}$, and oxygen saturation $64 \%$ which improved to $97 \%$ on the non-rebreather mask. Her physical examination was only significant for pain on palpation of the left calf with erythema, edema, and normal distal pulses.

Initial investigations revealed a complete blood count with hemoglobin $11.4 \mathrm{gm} / \mathrm{dl}$, white blood cell count 10.8/nL (80\% neutrophils, 10\% lymphocytes), platelet count 465/nL, prothrombin time (PT) $13.9 \mathrm{sec}$, international normalized ratio (INR) 1.2, partial thromboplastin time (PTT) 19.1 sec and D-dimer 1072 $\mathrm{ng} / \mathrm{mL}$, ferritin $536 \mathrm{ug} / \mathrm{L}$, C-reactive protein (CRP) $163.3 \mathrm{mg} / \mathrm{L}$. COVID-19 testing with reverse transcription polymerase chain reaction (RT-PCR) was positive. Chest X-ray showed bilateral ill-defined hazy infiltrates characteristic of COVID-19.

A computed tomography (CT) angiogram of the chest and lower extremity venous duplex was obtained due to high clinical suspicion for pulmonary embolism (Wells' score: 6, moderate risk group) and deep venous thrombosis (DVT) (Wells' score: 1, moderate risk group), respectively. Both the studies showed no evidence of a pulmonary embolus or DVT, however, CT angiogram of the chest captured a wedge-shaped area of low attenuation in the medial aspect of a normal-sized spleen, suggestive of a splenic infarct. 

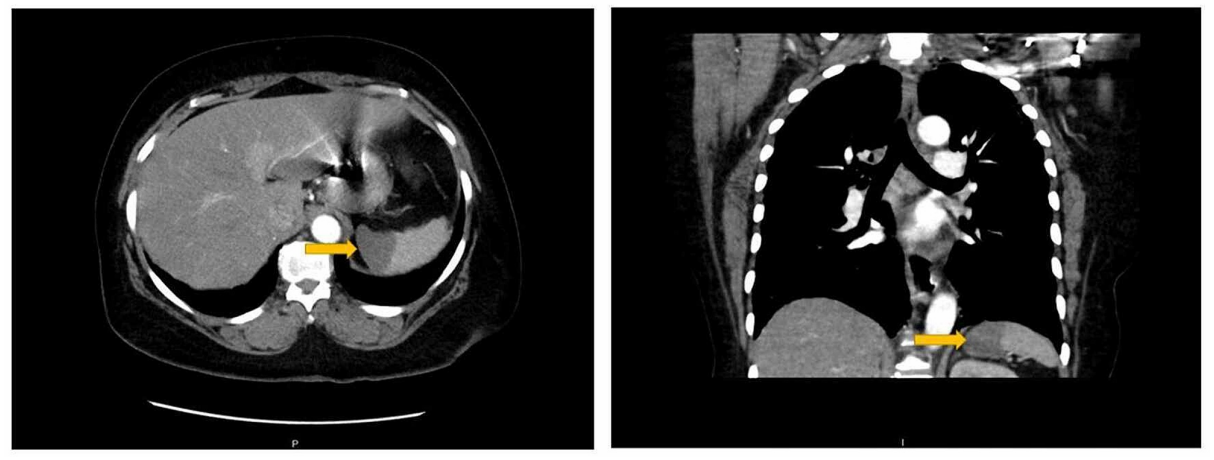

FIGURE 1: Axial and coronal view of the CT chest-abdomen showing area of low attenuation on the medial side of the spleen

A comparison to imaging from less than a month ago revealed a normal spleen on an ultrasound of the abdomen. Throughout her hospitalization, she denied abdominal pain, nausea or vomiting, and her abdominal examination remained benign.

The patient did not have any evidence suggestive of a cardio-embolic etiology for the splenic infarct. She did not have evidence of atrial fibrillation nor any stigmata of infectious endocarditis. Transthoracic echocardiogram did not show evidence of valvular lesions, right to left shunts or an intramural thrombus. Evaluation for anti-phospholipid syndrome showed a negative lupus anticoagulant, low positive cardiolipin immunoglobulin $\mathrm{G}(\mathrm{IgG})$ antibody ( $24 \mathrm{U} / \mathrm{ml})$, and a positive beta-2 glycoprotein 1 immunoglobulin $\mathrm{M}$ (IgM) antibody (25.6 SMU).

The patient was administered therapeutic anticoagulation with continuous heparin infusion. After starting anticoagulation, D-dimer decreased to $427 \mathrm{ng} / \mathrm{mL}$. After symptomatic management and resolution of hypoxia over seven days, the patient was discharged home on therapeutic anticoagulation with low molecular weight heparin (enoxaparin) with outpatient hematology follow up.

\section{Discussion}

COVID-19 is known to be a hypercoagulable state. Abnormal coagulation parameters, including elevated Ddimer, fibrinogen degradation products (FDP), low Factor XII levels, and positive lupus anticoagulant are well described in the literature to be associated with the risk for thrombosis [7-9]. Elevated D-dimer and FDP levels are associated with a poor prognosis [7,9]. There is a high incidence of venous thromboembolism, with most studies reporting an incidence between 20-27\% [2,5-6]. However, a French study reported rates as high as $69 \%$ in COVID-19 patients needing intensive care unit (ICU) level of care using a complete duplex ultrasound [10]. An Italian study reported a cumulative venous/arterial thromboembolic event rate of $6.6 \%$ in 314 patients admitted to the general medicine wards [6]. Amongst these, arterial thromboembolic events included stroke (1.9\%) and acute coronary syndrome (1\%) [6]. Another study on ICU patients in the Netherlands reported arterial thrombosis rate to be 3.7\% (all ischemic strokes) [5]. Except for these studies, data on the incidence of arterial thromboembolic events, especially in the splanchnic and peripheral vasculature, is scarce.

The presence of cardiolipin antibody and beta-2 glycoprotein 1 antibody could be a transient phenomenon in the setting of an acute infection. The elevated CRP does not predict the transient or persistent nature of these antibodies as shown by Twito et al. [11]. The patient needs re-testing after 12 weeks to establish the diagnosis of antiphospholipid syndrome. Zhang et al. recently described three cases with a negative lupus anticoagulant and positive cardiolipin and beta-2 glycoprotein 1 antibody in ICU patients with significant thrombotic disease, however, antibody titers were not mentioned in their report [12]. Since low titers are rarely associated with thromboembolism and are not considered in the Sapporo criteria [13], the relation of cardiolipin and beta 2 glycoprotein 1 antibodies with thrombo-embolic events in COVID-19 also needs further elucidation as we are dealing with a novel disease with potentially unknown pathological mechanisms. Endothelial inflammation from direct infection via viral entry through angiotensin-converting enzyme 2 (ACE-2) receptors present on endothelial cells has also been implicated in arterial thrombosis in COVID-19 patients [14].

In our case, the presence of a splenic infarct in the absence of a cardio-embolic source points to a primary arterial thromboembolic event in the splenic circulation due to COVID-19 related hypercoagulability. It adds to the evidence of a clinically silent arterial thrombotic event in a non-critical COVID-19 patient which further emphasizes the need to re-address the strategies for diagnosis and management of thromboembolism to prevent potentially fatal complications [15]. 


\section{Conclusions}

COVID-19 infection predisposes a hypercoagulable state with high incidence of both venous and arterial thrombosis. Splenic infarcts associated with COVID-19 have been rarely reported. A degree high of suspicion must be maintained in patients presenting with acute abdominal pain.

\section{Additional Information}

\section{Disclosures}

Human subjects: Consent was obtained or waived by all participants in this study. Conflicts of interest: In compliance with the ICMJE uniform disclosure form, all authors declare the following: Payment/services info: All authors have declared that no financial support was received from any organization for the submitted work. Financial relationships: Abhishek Kumar declare(s) stock/stock options from Bristol Meyer. Abhishek Kumar declare(s) stock/stock options from Bio Path holdings. Abhishek Kumar declare(s) stock/stock options from PFIZER. Abhishek Kumar declare(s) stock/stock options from POSEIDA Therapeutics. Abhishek Kumar declare(s) stock/stock options from BeyondSpring Inc. Abhishek Kumar declare(s) Stock/Stock Options from AIKIDO. Abhishek Kumar declare(s) stock/stock options from PTC therapeutics. Abhishek Kumar declare(s) stock/stock options from Cara Therapeutics. Abhishek Kumar declare(s) stock/stock options from ABBV. Abhishek Kumar declare(s) stock/stock options from ACADia Pharma. Abhishek Kumar declare(s) stock/stock options from AMGEN. Abhishek Kumar declare(s) stock/stock options from AVEO Pharma. Abhishek Kumar declare(s) stock/stock options from Astrazeneca. Abhishek Kumar declare(s) stock/stock options from Biotelemetry inc. Abhishek Kumar declare(s) stock/stock options from ADMA Biologics. Abhishek Kumar declare(s) stock/stock options from ChemBio Dagnostics. Abhishek Kumar declare(s) stock/stock options from AGNEUS. Abhishek Kumar declare(s) stock/stock options from Contrafect Corp. Abhishek Kumar declare(s) stock/stock options from Cardiff Oncology. Abhishek Kumar declare(s) stock/stock options from CRISPR Therapeutics. Abhishek Kumar declare(s) stock/stock options from CVS Health. Abhishek Kumar declare(s) stock/stock options from Precision Biosciences. Abhishek Kumar declare(s) stock/stock options from EDITAS Medicine Inc. Abhishek Kumar declare(s) stock/stock options from Five Prime Therapeutics. Abhishek Kumar declare(s) stock/stock options from Globus Medicine Inc. Abhishek Kumar declare(s) stock/stock options from IDEXX Laboratories. Abhishek Kumar declare(s) stock/stock options from Iovance Biosciences. Abhishek Kumar declare(s) stock/stock options from IDEXX Laboratories. Abhishek Kumar declare(s) stock/stock options from Iovance Biosciences. Abhishek Kumar declare(s) stock/stock options from Johnson and Johnson. Abhishek Kumar declare(s) stock/stock options from ELiLilly. Abhishek Kumar declare(s) stock/stock options from Northweast Bio. Abhishek Kumar declare(s) stock/stock options from Spectrum Pharmaceuticals iNc. Abhishek Kumar declare(s) stock/stock options from Viking Therapeutics. Abhishek Kumar declare(s) stock/stock options from Vertex Pharmaceuticals. Other relationships: All authors have declared that there are no other relationships or activities that could appear to have influenced the submitted work.

\section{References}

1. Richardson S, Hirsch JS, Narasimhan M, Crawford JM, McGinn T, Davidson KW, Northwell COVID-19 Research Consortium: Presenting characteristics, comorbidities, and outcomes among 5700 patients hospitalized with COVID-19 in the New York City area. JAMA. 2020, 323:2052-2059. 10.1001/jama.2020.6775

2. Terpos E, Ntanasis-Stathopoulos I, Elalamy I, et al.: Hematological findings and complications of COVID19. Am J Hematol. 2020, 95:834-847. 10.1002/ajh.25829

3. Jayarangaiah A, Theetha Kariyanna P, Chen X, Jayarangaiah A, Kumar A: COVID-19-associated coagulopathy: an exacerbated immunothrombosis response. Clin Appl Thromb Hemost. 2020, 26:10.1177/1076029620943293

4. Gupta N, Zhao YY, Evans CE: The stimulation of thrombosis by hypoxia . Thromb Res. 2019, 181:77-83. 10.1016/j.thromres.2019.07.013

5. Klok FA, Kruip M, van der Meer NJM, et al.: Incidence of thrombotic complications in critically ill ICU patients with COVID-19. Thromb Res. 2020, 191:145-147. 10.1016/j.thromres.2020.04.013

6. Lodigiani C, Iapichino G, Carenzo L, et al.: Venous and arterial thromboembolic complications in COVID-19 patients admitted to an academic hospital in Milan, Italy. Thromb Res. 2020, 191:9-14. 10.1016/j.thromres.2020.04.024

7. Tang N, Li D, Wang X, Sun Z: Abnormal coagulation parameters are associated with poor prognosis in patients with novel coronavirus pneumonia. J Thromb Haemost. 2020, 18:844-7. 10.1111/jth.14768

8. Bowles L, Platton S, Yartey N, et al.: Lupus anticoagulant and abnormal coagulation tests in patients with Covid-19. N Engl J Med. 2020, 383:288-290. 10.1056/NEJMc2013656

9. Zhang L, Yan X, Fan Q, Liu H, Liu X, Liu Z, Zhang Z: D-dimer levels on admission to predict in-hospital mortality in patients with Covid-19. J Thromb Haemost. 2020, 18:1324-1329. 10.1111/jth.14859

10. Llitjos JF, Leclerc M, Chochois C, Monsallier J-M, Ramakers M, Auvray M, Merouani K: High incidence of venous thromboembolic events in anticoagulated severe COVID-19 patients. J Thromb Haemost. 2020, 18:1743-1746. 10.1111/jth.14869

11. Twito O, Reshef T, Ellis MH: C-reactive protein level as a predictor of transient vs. sustained anticardiolipin antibody positivity. Eur J Haematol. 2006, 76:206-9. 10.1111/j.1600-0609.2005.00592.x

12. Zhang Y, Xiao M, Zhang S, et al.: Coagulopathy and antiphospholipid antibodies in patients with Covid-19. N Engl J Med. 2020, 382:38. 10.1056/NEJMc2007575

13. Miyakis S, Lockshin MD, Atsumi T, et al.: International consensus statement on an update of the 


\section{Cureus}

classification criteria for definite antiphospholipid syndrome (APS). J Thromb Haemost. 2006, 4:295-306.

10.1111/j.1538-7836.2006.01753.x

14. Varga Z, Flammer AJ, Steiger P, et al.: Endothelial cell infection and endotheliitis in COVID-19. Lancet. 2020, 395:1417-1418. 10.1016/S0140-6736(20)30937-5

15. Cannegieter SC, Klok FA: COVID-19 associated coagulopathy and thromboembolic disease: commentary on an interim expert guidance. Res Pract Thromb Haemost. 2020, 4:439-445. 10.1002/rth2.12350 